\title{
Invited review: Measurable biomarkers linked to meat quality from different pig production systems
}

\author{
Marinus F. W. te Pas ${ }^{1}$, Bénédicte Lebret ${ }^{2}$, and Niels Oksbjerg ${ }^{3}$ \\ ${ }^{1}$ Wageningen UR Animal Breeding and Genomics, Wageningen, the Netherlands \\ ${ }^{2}$ INRA, French National Institute for Agricultural Research, UMR1348 PEGASE, 35590 Saint-Gilles, France \\ ${ }^{3}$ Aarhus University, Dept. of Food Science, Faculty of Agricultural Sciences, Dept. of Food Science, \\ P.O. Box 50, 8830 Tjele, Denmark \\ Correspondence to: Marinus F. W. te Pas (marinus.tepas@wur.nl)
}

Received: 27 March 2017 - Revised: 16 June 2017 - Accepted: 26 June 2017 - Published: 10 August 2017

\begin{abstract}
Biological processes underlie all livestock traits, including post-mortem meat quality traits. Biomarkers are molecular components of the biological processes showing differential expression associated with the phenotype of the trait. The phenotypes of the meat quality traits are determined by the animal's genotype interacting with the environment affecting the expression of the genome. The "omics" technologies enable measuring the expression of the genome at all levels: transcriptome, proteome, and metabolome. Associations between the phenotype of the traits and expressions measured with the omics techniques are a first step in developing biomarkers. Biomarkers enable the monitoring, diagnosis, and prediction of changes in meat quality related to external (environmental, e.g. feed and animal management conditions) stimuli and interactions with the genotype. In this paper we review the development of biomarkers for meat quality of pigs in diverse pig breeds, environments, and pork production chains.
\end{abstract}

\section{Introduction}

The aim of this paper is to review results of research aiming to develop biomarkers for meat quality. Specific emphasis will be given to biomarkers of fresh pork and processed pork products, even though provision of biomarkers for meat quality management is a current topic of high interest in all livestock species, including fish (Picard et al., 2012, 2015). We will describe the use of omics technologies, bioinformatics, and systems biology for biomarker development. Meat quality is among the most important characteristics of meat for consumers. Sensory quality traits, including meat appearance (colour, marbling, etc.) and texture (taste, tenderness, juiciness, and chewiness), as well as flavour and taste, are most appreciated. Suitability for different preparatory methods by the processing industry and shelf life (storability, colour, and stability) are accompanying meat quality components (Lebret and Picard, 2015). Since muscle tissue becomes meat after slaughtering, the composition and metabolic properties of muscle tissue are among the most important factors in- fluencing meat quality, together with the animal's management before slaughtering and post-mortem carcass management (Warriss, 2010).

Muscle is a complex tissue composed of several cell or tissue types, including muscle fibres of different types, adipose cells, neural cells, endothelial cells, and connective tissue (Bailey and Light, 1989; Gandemer, 2002; Purslow, 2005; Listrat et al., 2016). Muscle fibre types include type I, IIA, IIB, and IIX (Lefaucheur, 2010). These types differ in metabolic and contractile properties: type I exhibits oxidative metabolism and slow contraction speed, whereas type II fibres are fast fibres and exhibit oxydo-glycolytic (IIA and IIX) or glycolytic metabolism (IIB). Myofibre typing directly determines not only the meat quality trait colour but also the in vivo and post-mortem muscle energy metabolism, which has a major impact on technological and sensory quality traits (Henckel et al., 2000; Listrat et al., 2016). Among other things, the energy content of the muscle fibres - e.g. the glycogen content stored in the muscle fibres (Milan et al., 2000; Ciobanu et al., 2001; Rosenvold et al., 2001) - greatly 
influences the post-mortem processes important for the development of meat quality. Furthermore, type IIB fibres are generally thicker than type I fibres. The cross-sectional area (CSA) of type IIB is 2-fold larger than type I and type IIA. The CSA of the latter two is similar; this histological property is related to drip loss, an important meat quality determinant (Lefaucheur, 2010). Adipose tissue, both from intermuscular fat and intramuscular fat, is an important factor for meat taste (Cameron and Enser, 1991; Fernandez et al., 1999), but too much of it is not appreciated by consumers: generally 2.5-3\% intramuscular fat is considered optimal (Listrat et al., 2016). Thus, meat quality is a complex trait affected by multiple components and moreover can be fully determined only a few days after slaughter when the ageing processes have occurred. Predicting the potential of meat for a certain quality shortly after, or even before, slaughter of the animal is therefore a challenging task. Biomarkers are tools that can be helpful for this.

At present meat quality is determined after ripening of the meat either by a trained taste panel or by measuring objective technical parameters (Hovenier et al., 1993; Honikel, 1998). While a sensory-trained panel "measures" "sensory" meat quality traits such as appearance, texture, and taste, technological meat quality traits relate to the ability of the fresh meat to be processed and includes technological or cooking yields such as colour, drip loss, and fat content. Of particular note, sensory and technological meat quality traits are intertwined and depend on the same post-mortem processes. All of these quality dimensions are determined by interactions between animal genetic background, rearing, and slaughtering conditions and meat processing factors and are related to the biological processes underlying the post-mortem processes of transformation of muscle into meat. Knowing the biological processes involved in the development of meat quality potentially opens the possibility to monitor, predict, and interfere with the final meat quality. Biomarkers often are molecules involved in one or more of the biological processes underlying a specific meat quality trait (discussed in te Pas et al., 2011a, and te Pas and Hoekman, 2012). Because they are related to or participate in the biological processes, they can be used to monitor these processes. More importantly, based upon pre-existing knowledge of the relation between the biomarker and the biological processes, biomarkers can also predict the outcome of the post-mortem processes - and therefore predict the final meat quality before it has fully developed, or even before slaughter. This opens the potential to manage the final meat quality and the (global) meat quality in the chain, e.g. select carcasses or cuts for specific uses such as dry-cured ham or sausage production according to their intrinsic characteristics and potential quality value, and thereby improve added value for the pork industry.

Specific meat products such as local products or products as dry-cured hams may require specifically selected livestock or animal management (Bonneau and Lebret, 2010). This may affect the above-mentioned biological processes. Therefore, biomarkers may be specific for production systems. In this review we will focus on fresh pork production from commercial lines (a German and a Danish production chain), local production lines (a local breed production chain from France), and specific products (a dry-cured ham production chain from Spain). We will also focus on specific aspects (e.g. prenatal muscle fibre development, and pre-slaughter transport stress) of meat quality. In the discussion we will integrate the knowledge and discuss the possibilities for (commercial) use of the biomarkers.

\section{The concept of biomarkers for meat quality}

The concept of biomarkers for meat quality has been discussed before (te Pas et al., 2011a; te Pas and Hoekman, 2012) and here we refer to this discussion briefly. The breeding industry is increasingly using genetic markers to improve the genetic potential of livestock through genomic selection (Calus and Veerkamp, 2007). While this is important for the production chain as a whole, meat quality is a phenotype. The other industries in the pork production chain focus on producing the best phenotype related to the genotype produced by the breeding industry. Due to the influence of the environment of animals on the determination of production traits (phenotype) the use of genetic markers is not sufficient. Biomarkers also take these environmental effects into consideration by detecting the expression of the genome, which is the combination of genetic potential and environmental modulation (Goodsaid and Frueh, 2007).

Biomarkers are molecular components of biological processes regulating phenotypes of animals, including meat quality. Different phenotypes may be regulated by differences in (part of) the biological processes underlying the phenotype. Biomarkers are derived from improved knowledge of the biological mechanism underlying economically interesting and complex phenotypic traits such as meat quality. Biomarkers are frequently but not necessarily part of the biological mechanism, and often they refer to molecules or the level (concentration) of molecules. Biomarkers may be molecules of any type (e.g. RNA, proteins, metabolites) or may consist of a profile of several molecules (te Pas et al., 2011a; te Pas and Hoekman, 2012; Picard et al., 2015). Frequently, the level of the biomarker is associated with quantitative aspects of the trait (Wilson and De la Vega, 2005; te Pas et al., 2010).

To explain the concept of biomarkers we use a well-known example: blood glucose level as a biomarker for diabetes type 2. Blood glucose level can be used by a physician to determine whether a patient has (diagnosis), or is developing (prediction), diabetes and by the patient to determine how much insulin to use (monitoring). The causal factor for the diabetes is a lack of insulin or insulin resistance (for a review see Tisch and McDevitt, 1996). It is possible to detect insulin 
hormone levels in blood (Phillips et al., 1994) and to measure functional hormone binding to its receptor (Gammeltoft, 1984), but these tools require skilled laboratory technicians and are therefore time-consuming and costly. Knowing the biological mechanism of insulin shows that the biological action of insulin is to import glucose from the blood into cells, as a consequence regulating the blood glucose level. It is easy, fast, and inexpensive to measure blood glucose level. Furthermore, automation makes it possible for diabetes patients to measure blood glucose themselves at low cost. Thus, blood glucose level measurement is the biomarker of choice for predicting and diagnosing diabetes. Furthermore, the measured glucose level can be used for intervention: injection of insulin when blood glucose is too high, or glucose intake if blood glucose is too low. This example shows how knowledge of the biological process leads to an effective biomarker. Another example may be biomarkers related to gestation. During gestation, rapid changes in gene expression as well as hormone profiles occur. This illustrates that biomarkers can change rapidly throughout life. This is in contrast with genetic markers that are stable in the genome throughout life.

From this it may be concluded that once the biological mechanism underlying a trait is known, there are many potential biomarkers available (although biomarkers with unknown functionality do exist). The question is how to choose between them. Characteristics of good biomarkers are as follows: (1) a high predictive value for the trait, (2) easy and cheap detection (i.e. lower than the economic value of the trait) (Goodsaid and Frueh, 2007) and (3) measurability during the production phase using non-invasive or easy and cheap sampling methods of tissues such as blood or excreted body fluids. Even when variability in the trait between animals is low, the biomarker should remain specific for the trait and preferably constant over a certain time period. For large-scale testing, automation of testing combined with information technology may speed up the industrial procedures and thereby reduce the costs of production in industries.

\subsection{How to develop biomarkers: the use of "omics" methods and analysis tools}

Biological processes are regulated at diverse levels. Mutations at the genomic DNA level may affect the functionality of proteins, or affect the expression of the genes. The expression of the genes is also regulated by differences in methylation of the DNA, the activity of non-coding small RNA molecules such as miRNAs, the activities of diverse transcription factors, and the activity of the translation components of the cell (Kadonaga, 1998; Li et al., 2013). Furthermore, the activity of the proteins is further regulated by post-translational modifications regulating the flux through biological mechanisms, potentially differentially regulating metabolite concentrations in the cell.
High-throughput omics techniques can measure all of these biological levels: genomics measures single nucleotide polymorphisms (SNPs) related to genetic variation, leading to the development of genetic markers; transcriptomics measures RNA and miRNA levels related to the activity of the genome; proteomics measures protein levels and posttranslational modifications related to the biological functionality of the genome; and metabolomics measures metabolite levels related to the biological processes themselves, leading to the development of biomarkers. Together these tools make a full description of all the biological mechanisms underlying a trait. Bioinformatics is necessary to decipher the underlying networks and pathways (i.e. how genes cooperate) and the functional biological processes (te Pas et al., 2006, $2007 a, b)$. Systems biology integrates the knowledge of all levels in complex biological models (Woelders et al., 2011; te Pas et al., 2011b). Ideally, knowledge of all these underlying mechanisms should be available, but usually biomarkers are developed on the knowledge of only one or two of the omics methods. This may be the cause of different biomarkers using the same samples. Since traits like meat quality characteristics are multi-gene complex traits, this can be expected.

Often a very important prerequisite for developing biomarkers is overlooked: the availability of (highly) contrasting phenotypes including also large datasets with gradual but great variability in meat quality parameters. These quality phenotypes should be measured with conventional methods such as Minolta (colour) and $\mathrm{pH}$ at 1 and $24 \mathrm{~h}$ (related to many meat quality traits such as the water-holding capacity and the lightness of the meat) and determined, for example, by muscle energy metabolism and buffering power, glycogen content at time of slaughter, drip loss, intramuscular fat content (IMF) (the last two both related to tenderness and juiciness), shear force, sensory tenderness, juiciness, and flavour by a trained panel (Honikel, 1998). Combining the information from omics studies and phenotype information using bioinformatics and statistics (a so-called association study) can highlight the relationship between the two. From this information, biomarkers can be identified. Below we will provide several examples of this from a variety of pork production chains and using various omics methodologies.

\section{Description of existing biomarkers}

In this section we summarize published results of investigations aiming to develop biomarkers for pork quality. We will show how different pork production chains were investigated with different omics methods, highlighting the biology underlying the traits leading to different biomarkers. The results originate from various projects including industry and publicly funded projects. Most of these projects were in cooperation with the breeding industry, where there is widespread interest in genetic and biomarkers (Wilson and De la Vega, 2005). This seems logical since the breeding industry also 
recognizes the effect of the interactions between genotype and environment in the determination of phenotypic traits such as meat quality. Furthermore, the increased use of the SNP chip and the positive results may pave the way for the use of expressional biomarkers by other industries in the pork production chain.

\subsection{Biomarkers for high-quality fresh pork production}

While a major part of the world pork market is comprised of fresh meat, a wide variety of processed products (either cured or cooked, and prepared from entire cuts or minced/mixed pieces) are consumed. For example, in France, more than $75 \%$ of pork is consumed as processed products. Therefore the requirements of the pork and the expectations of the consumers vary widely according to the final product considered and also to the geographical origin of the consumers, not only throughout the world but also in closely related areas such as the EU. Therefore, a large variety of pig breeds and pork production chains exist. We used a German high-quality fresh pork production chain based on Pietrain pigs. This breed is known for high dressing percentage and high meat percentage but is also known to suffer from porcine stress syndrome (PSS), leading to sudden death and pale-coloured meat with a high initial rate of $\mathrm{pH}$ decrease, leading to high drip loss (Kukoyi et al., 1981). Because of its high dressing percentage and very lean meat, the breed is popular for fresh pork production in certain areas of Europe.

We used a German selection line for high fresh meat quality measured as low drip loss, non-pale meat colour (even redder meat than usual for the Pietrain breed), and a related relative slow drop of (initial) $\mathrm{pH}$ post-mortem, and we sampled carcasses derived from the offspring of Pietrain boars deemed either high fresh meat quality or average meat quality (te Pas et al., 2010). The contrast in transcriptomic profiles between the two types of carcasses was studied. Because the focus of the study was directed to the general biological differences between the two groups of animals rather than to the individual differences within a group, the samples from each group were pooled. Because the meat quality measurements were on the longissimus dorsi, this muscle was also used to study the transcriptome with microarray technology.

The results show that the genes differentially expressed between the two groups carcasses were related to musclespecific processes such as contraction and contractile fibre type, oxygen transport, and intracellular processes. The association study highlighted the importance of muscle colour, drip loss, and rate of $\mathrm{pH}$ decrease ultimate $\mathrm{pH}$ (i.e. the end $\mathrm{pH}$ of the meat, usually measured at $24 \mathrm{~h}$ after slaughtering; see Honikel, 1998) together with carcass traits (te Pas et al., 2010, summarized in Table 1). The results suggest that in this Pietrain line high-quality pork relates to the redness of the meat, reduced drip loss, and the rate of $\mathrm{pH}$ decrease and ultimate $\mathrm{pH}$. These meat quality changes also relate to carcass traits. Due to the specific characteristics of the Pietrain pigs,
Table 1. Number of biomarkers found per trait in a German highquality fresh pork Pietrain selection line.

\begin{tabular}{|c|c|}
\hline Trait & Number of biomarkers \\
\hline $\mathrm{a}^{*}$ value $^{\mathrm{a}}$ & 14 \\
\hline Reflection & 10 \\
\hline Drip loss $(\%)$ & 2 \\
\hline $\mathrm{pH} 1^{\mathrm{b}}$ & 1 \\
\hline $\mathrm{pH} 3^{\mathrm{b}}$ & 1 \\
\hline $\mathrm{pH} 6^{\mathrm{b}}$ & 1 \\
\hline $\mathrm{pH} 24^{\mathrm{b}}$ & 6 \\
\hline Back fat thickness $(\mathrm{mm})$ & 4 \\
\hline Carcass weight $(\mathrm{kg})$ & 4 \\
\hline Meat thickness (mm) & 2 \\
\hline Lean meat $(\%)$ & 3 \\
\hline
\end{tabular}

i.e. high stress susceptibility related to pale and exudative meat, it is interesting that high-quality meat carcasses seem to be selected especially for colour redness and reduced drip loss and related $\mathrm{pH}$ traits.

Ten of these genes were studied in a validation study using a PCR test for the genes (Pierzchala et al., 2014). In this validation study, 100 Danish commercial Large White pigs with all phenotypic data available were used. It is always important to validate the results in an independent group of samples, if possible with a different genetic background. One difficulty in such studies may be that technical meat quality traits are measured differently. This may cause variation in the data. The PCR results were used to build a model to explain the contribution of each of the genes to each of the traits. The results confirmed the Pietrain microarray data and found in addition associations for other quality traits such as colour and IMF as well. The modelling showed that the highest explanation of the phenotypic variance of a given quality trait in a best fit model was found for ultimate $\mathrm{pH}$ at $55 \%$, followed by the meat colour traits. Since this validation study did not include all genes of the microarray study, the biomarker composed of all genes reported in the first study would probably have a higher explanation of the variation in the traits.

\subsection{Biomarkers for resources for high-quality dry-cured ham production}

Dry-cured ham is a high-value pork product. During production the hams are prepared using a special procedure (depending upon the region where the hams are produced), after which the hams are dried for between a few months and several years (Callow, 1947; Meats and Sausages, 2017; Wierbicki et al., 1976; Zhou and Zhao, 2007). The aroma of drycured ham originates mostly from proteolysis and lipolysis, and while lipolysis is affected primarily by manufacturing 
temperature, proteolysis is inhibited by salt (Toldra et al., 1997; Toldra and Flores, 1998). Since the preparation procedure requires a ham with minimum subcutaneous fat, a terminal Duroc boar is used and the average slaughter weight of the pigs is $120-140 \mathrm{~kg}$. Thus, additional animal management and slaughter costs appear. Only after finishing the premortem and post-mortem procedures can the quality of the dry-cured ham be determined. It is clear that a low-quality final product has high costs for the whole production chain without related income raised from the product. Thus, the use of high-quality green hams is important. Biomarkers can be used to recognize the best-quality green hams to avoid further quality problems and high costs without revenues. But the quality of the green ham needs to be determined using non-invasive methods to avoid any damages to the product. Since the muscles in an animal relate to each other to a certain degree, the meat quality characteristics can be studied in the longissimus dorsi muscle and extrapolated to the ham.

The aim of the study was to find biomarkers for dry-cured ham quality. We used proteomics because we expected that the cytosolic protein levels in the different muscles may be more related to each other than the transcriptomics expression profiles: indeed, Herault et al. (2014) showed great differences between semimembranosus and longissimus gene expression profiles. Proteomics was studied using two methods and in two independent batches of animals originating from two different farms. Although these animals were genetically related we used them as comparisons for each other without further validation using different breeds. Previous studies with different breeds showed that breed and muscle type did not affect the number of peaks per spectrum in SELDI-TOF experiments, while a few of these peaks indicated muscle fibre type-specific proteins (Mach et al., 2010). Marcos et al. (2013a, b), comparing four different pure breeds (Duroc, Large White, Landrace, and Pietrain) using the same technique, showed associations between protein peaks and meat quality traits, mainly water-holding capacity and texture. Although explaining a limited proportion of the variability, the authors concluded that the results showed interesting relationships between protein expression and longissimus meat quality traits. Furthermore, using the same four breeds the ham quality for dry-cured ham production was studied by these authors. Surprisingly, they concluded that even if differences in the quality traits (colour, water activity, texture, composition, IMF, and marbling) of dry-cured hams were observed among the studied breeds, only small differences in the sensory attributes were detected. However, associations between protein peaks and meat quality traits, mainly colour (Minolta $b^{*}$, meat yellowness) and texture (for details see Marcos et al., 2013a, b), were observed. Candidate protein markers for the quality of processed dry-cured hams were identified (Marcos et al., 2013b).

Peak profile proteomics analysis revealed that most peaks were shared by all carcasses (te Pas et al., 2013). This is of importance because the samples were taken after hanging the carcasses overnight, and previous results have shown differences between proteins in post-mortem protein degradation profiles and therefore a potential influence of sampling time on proteomic profile (te Pas et al., 2009). Statistical association studies revealed biomarkers for a number of traits, including drip loss, ultimate $\mathrm{pH}$, colour, and fatness traits. Biomarker profiles for ultimate $\mathrm{pH}$ and drip loss showed the highest predictive capacity, with a maximum of over $80 \%$. The number of proteins included in the biomarker differed per trait. For IMF only a single protein was included, similar to previous studies (Gerbens et al., 1997, 1998, 1999, 2000, 2001; Damon et al., 2006) reporting a large effect of the fatty acid binding protein (FABP) genes and the abundance of protein FABP4 on IMF depot regulation. For drip loss 5 proteins were included and up to 20 proteins included for ultimate pH (te Pas et al., 2013). It is important to note that a good biomarker can be composed of a combination of (different types of) molecules. Further proteomics analysis identified the important proteins in the profiles and biological analyses of the important traits drip loss and ultimate $\mathrm{pH}$ showed that several of the proteins found simultaneously were involved in the determination of both traits, in agreement with the high correlation generally found between these two quality traits. This illustrates the complexity of quality traits and shows why the optimal number of proteins in the biomarkers may be high for several traits.

The results are also suggestive of the underlying biological mechanisms of muscle transformation into meat and development of quality traits: therefore, knowing the biomarkers bring understanding of why the traits are complex and related to each other. A suggestive biological mechanism may be that, after slaughtering, protein degradation occurs. As a result the cells become leaky, leading to a loss of water from the tissue: drip loss. The amount of protein degradation may relate to the amount of energy stored in the muscle affecting both the ultimate $\mathrm{pH}$ and the amount of drip loss. However, Of particular note is that the real biochemical post-mortem processes may be much more complex than this.

\subsection{Biomarkers for high-quality meat from a local breed}

As mentioned above, eating and technological qualities of pork result from interactions between genetic and environmental factors; even though many factors influencing pork quality have been identified, its variability remains high and the muscle properties underlying high eating quality are still unclear (Ngapo and Gariépy, 2008). In order to decipher the biological mechanisms leading to high-sensory pork and identify biomarkers of high-quality meat, we took advantage of the development of genomic studies to analyse the transcriptomic profile of two contrasting breeds, the Large White (LW), considered as conventional with satisfactory quality pork, and the French local Basque (B) breed, leading to high sensory and technological quality of fresh pork 
and processed products (Bonneau and Lebret, 2010). Transcriptome analysis of the longissimus muscle of $20 \mathrm{LW}$ and $20 \mathrm{~B}$ pigs was undertaken using a custom $15 \mathrm{~K}$ microarray, corresponding to around 9000 unique genes. This study highlighted 1233 genes differentially expressed between breeds (multiple-test adjusted $P$ value $<0.05$; Benjamini and Hochberg, 1995), out of which 635 were highly expressed in the B and 598 highly expressed in the LW pigs. Functional annotation clustering emphasized four main clusters associated with transcriptome breed differences: metabolic processes, skeletal muscle structure and organization, extracellular matrix, lysosome, and proteolysis, thereby highlighting many genes involved in muscle physiology and meat quality development (Damon et al., 2012).

In order to get a progressive and high variability in pork quality within our data set, these two contrasted breeds were produced in different production systems, themselves influencing pork quality: conventional, alternative (litter bedding and outdoor access), and extensive system of the Basque pork chain (B pigs only) (Lebret et al., 2015). Within both $\mathrm{B}$ and LW pigs, the alternative system did not influence the transcriptomic profile and the quality traits of fresh pork. However, within the B breed, the extensive system led to higher $\mathrm{pH} 30 \mathrm{~min}$ post-mortem (slower post-mortem $\mathrm{pH}$ fall) and ultimate $\mathrm{pH}$, darker meat and better water-holding capacity (lower drip loss) but slightly higher shear force (Lebret et al., 2015). These quality differences between production systems were associated with differences in the longissimus transcriptome profile: using the same microarray as described above, we highlighted up-regulation of 117 genes in the longissimus muscle of B pigs reared in the extensive system and 150 genes up-regulated in B pigs from the conventional or alternative production systems. Functional annotation clustering of biological processes associated with these transcriptome differences emphasized eight main clusters, in particular genes involved in the control of muscle structure and thermal response (small heart shock proteins (HSPs)) in pigs reared in the extensive compared with the alternative or conventional systems (Lebret et al., 2013a). In addition to the analysis of the influence of breed and of pig production system on the muscle transcriptome profile by contrasted analysis, the wide range and progressive variability in technological and sensory quality of pork as well as of muscle expression patterns obtained in our experimental design were exploited to identify biomarkers for pork quality (ultimate $\mathrm{pH}$; colour: lightness, redness, hue angle; drip loss; IMF content; shear force; sensory tenderness; juiciness; and flavour) (Fig. 1). Combining meat quality and transcriptomic data obtained on 50 animals, we established thousands of correlations between microarray gene expression and meat quality traits (140 for Minolta a* (meat redness) up to 2892 for tenderness, adjusted Benjamini and Hochberg $P$ value $<0.10$ ) (Damon et al., 2013). Then, considering 40 genes selected for high correlation coefficient values or relevant biological process terms for meat quality development, these associa- tions were confirmed on the same samples using RT-PCR technique, which is more accurate for the further development of molecular tools. Thus, 113 transcript-trait associations were confirmed $\left(P<0.05, R^{2} \leq 0.52\right)$, out of which 60 were validated $\left(P<0.05, R^{2} \leq 0.46\right)$ on complementary data from the same experiment $(n=50)$ (Table 2). In other words, this means that the expression level of one gene could explain up to $46 \%$ of the variation of one quality trait, in the present case expression of ANKRDI (Ankyrin repeat domain 1) as a biomarker of ultimate $\mathrm{pH}$. These correlations involved a total of 26 genes and 8 meat quality traits. Moreover, multiple regression models including three to five genes explained up to $59 \%$ of the phenotypic variability in meat quality, with best models (highest $R^{2}$ ) found for meat colour (hue angle), ultimate $\mathrm{pH}$, drip loss, and IMF (Damon et al., 2013). Finally, an external validation was undertaken using pigs from different genetic origin produced in a commercial chain $(n=100$ commercial Duroc $\times$ Landrace $\times$ Yorkshire pigs) and allowed validating 19 transcript-trait associations $\left(R^{2} \leq 0.24, P<0.05\right)$, i.e. biomarkers for ultimate $\mathrm{pH}(6)$, drip loss (4), Minolta $\mathrm{L}^{*}$ (meat lightness) (5), hue angle $\left(\mathrm{h}^{\circ}\right)$ (2), IMF content (1), and tenderness (1) (Table 2) (Lebret et al., 2013b).

Altogether, these results show that biomarkers of pork eating and sensory quality have been identified and validated, but further work is needed to improve their predictive capacity in order to predict the development of control tools for pork industry. Therefore, another approach based on the identification and validation of transcriptomic biomarkers of sensory and technological meat quality grades (e.g. low, acceptable, or extra pork quality levels) has been recently developed (Lebret et al., 2014). The final aim is to propose molecular tools allowing discrimination of carcasses or primary cuts early after slaughter according to their predicted sensory or technological quality level.

\subsection{Biomarkers for detection of meat quality in relation to transport stress and recovery from transport stress}

Pre-slaughter stress, for example transport exercise, is an important factor influencing meat quality. Exercise changes muscle metabolism and related muscle composition, especially in untrained muscles. Pre-slaughter pig transportation can be regarded as exercise of untrained muscles. However, rest after the exercise may reverse (most of) the effects of exercise on meat quality. Pre-slaughter exercise and time of rest between exercise and slaughter may affect meat quality traits differently (Grandin, 1980; Van der Wal et al., 1999; Støier et al., 2001; Kuchenmeister et al., 2002, 2005; Schäfer et al., 2002) because the underlying molecular mechanisms differ between traits. In a model study for transportation exercise and stress, pigs were placed on a treadmill for $30 \mathrm{~min}$. The pigs were slaughtered either immediately after the exercise or after 1 or $3 \mathrm{~h}$ of resting time (Young et al., 2009). 


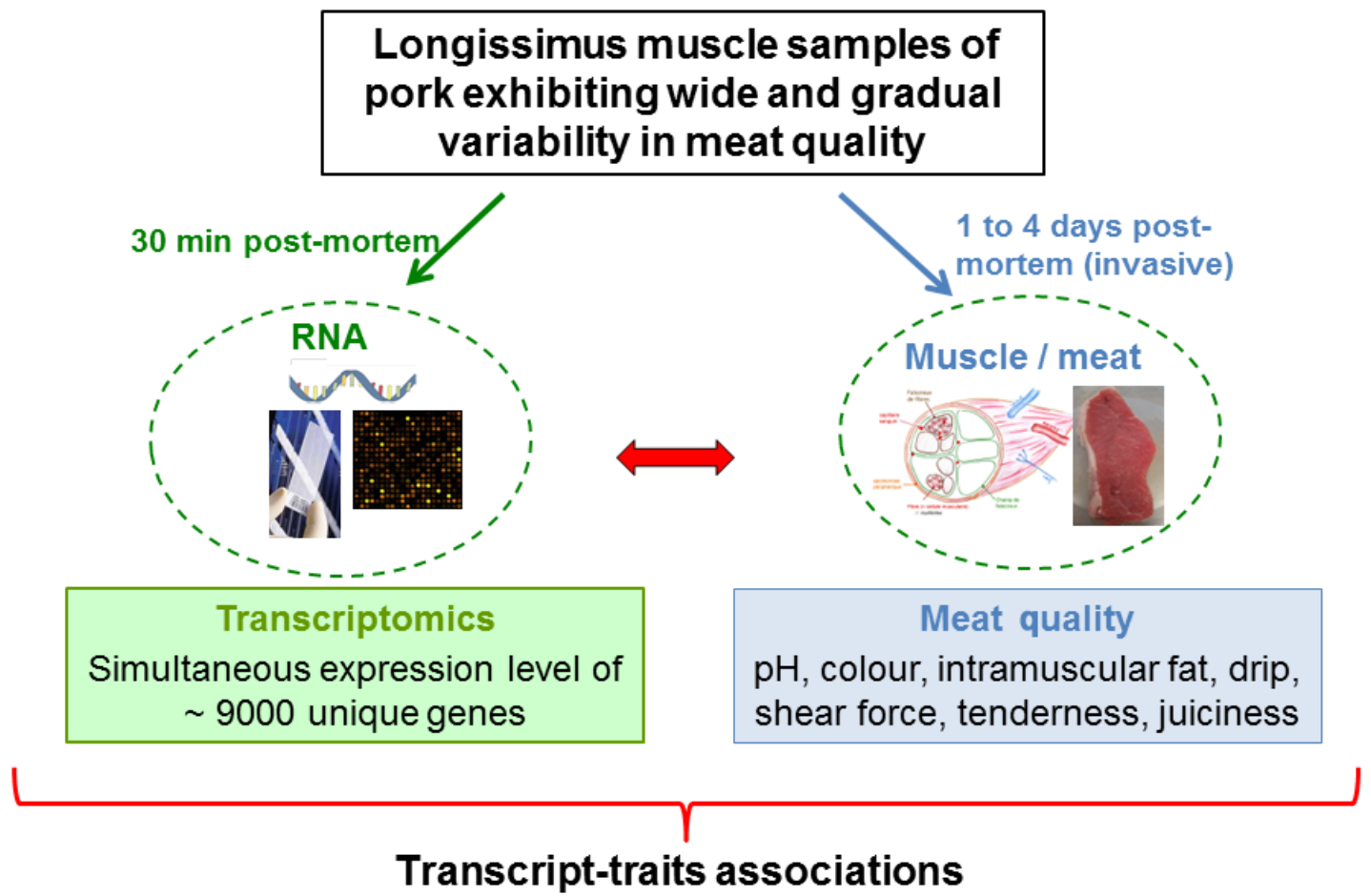

Figure 1. Identification of biomarkers of meat quality by transcriptomic analysis of longissimus muscle: association between gene expression level on muscle $15 \mathrm{~K}$ microarray and individual meat quality traits (ultimate $\mathrm{pH}$; colour: lightness, redness, hue angle; drip loss; intramuscular fat content; shear force of cooked meat; sensory tenderness; and juiciness).

Table 2. Identification, confirmation and validation of biomarkers of pork quality. Significant correlations $(P<0.05)$ between meat quality traits and the expression level of 40 genes at steps of identification (microarray, $n=50$ pigs), confirmation (RT-PCR, $n=50$ same pigs as identification step), internal validation ( $n=50$ different animals from same experimental design as confirmation step), and external validation (RT-PCR, $n=100$ pigs genetically different from previous steps, commercial chain). Adapted from Lebret et al. (2013b).

\begin{tabular}{|c|c|c|c|c|}
\hline Quality trait & Identification $^{\mathrm{a}}$ & Confirmation $^{\mathrm{a}}$ & Internal validation $^{\mathrm{a}}$ & External validation $^{\mathrm{a}}$ \\
\hline Ultimate $\mathrm{pH}$ & $15(0.65)$ & $12(0.60)$ & $10(0.68)$ & $6(0.49)$ \\
\hline Drip loss $(\%)$ & $24(0.72)$ & $23(0.58)$ & $14(0.51)$ & $4(0.34)$ \\
\hline Meat lightness $\left(\mathrm{L}^{*}\right)$ & $18(0.56)$ & $16(0.50)$ & $11(0.55)$ & $5(0.28)$ \\
\hline Meat redness $\left(\mathrm{a}^{*}\right)$ & $7(0.63)$ & $7(0.59)$ & $4(0.39)$ & 0 \\
\hline Hue angle $\left(\mathrm{h}^{\circ}\right)$ & $18(0.59)$ & $16(0.61)$ & $10(0.62)$ & $2(0.24)$ \\
\hline $\mathrm{IMF}^{\mathrm{b}}$ & $17(0.72)$ & $14(0.60)$ & $6(0.47)$ & $1(0.24)$ \\
\hline Shear force $\left(\mathrm{N} \mathrm{cm}^{-2}\right)$ & $9(0.69)$ & $7(0.46)$ & $1(0.29)$ & $\mathrm{Nd}^{\mathrm{c}}$ \\
\hline Tenderness & $20(0.80)$ & $18(0.73)$ & $4(0.37)$ & $1(0.22)$ \\
\hline
\end{tabular}

${ }^{\mathrm{a}}$ Number of significant correlations and, in brackets, maximum absolute value of correlation coefficient $(|r|)$. ${ }^{\mathrm{b}}$ IMF: intramuscular fat content. ${ }^{\mathrm{c}}$ Not determined.

Exercise increased the muscle temperature by about $1{ }^{\circ} \mathrm{C}$ in the longissimus dorsi and $2{ }^{\circ} \mathrm{C}$ in the biceps femoris, which was reversed within $1 \mathrm{~h}$. This showed the (high) intensity level of the exercise and the functional differences of the muscles (respectively static and active during the exercise). In both muscles, creatine phosphate, ATP, and glycogen content were reduced, and $1 \mathrm{~h}$ of resting also reverses these biochemical effects. These biochemical changes affected technical meat quality traits (reduced the rate of early post-mortem
$\mathrm{pH}$ decrease and increased drip loss). The authors reported overshooting effects for the $3 \mathrm{~h}$ resting period for $\mathrm{pH}$ and drip loss in the biceps femoris muscle and the primary energy sources (creatine phosphate and ATP). Furthermore, exercise increased the toughness of the meat, which was not reversed by resting for 1 or $3 \mathrm{~h}$ (Young et al., 2009). Because the biochemical parameters were reversed by resting this toughness seems not to be related to drip loss. 
The resulting biochemical changes affect proteome muscle composition and biochemical processes related to energy metabolism, which also affect post-mortem processes affecting meat quality. We studied the proteome changes in pig longissimus dorsi and biceps femoris muscle tissue of the same animals (te Pas et al., 2011c). The results show that the expression levels of many proteins in the muscle proteome are affected by both exercise and resting, and the muscle proteome of both muscles reacted differently to the applied exercise stress, probably due to the fact that the biceps femoris is directly involved in running while the longissimus dorsi muscle is not. Many of the observed associations relate to muscle energy metabolism and storage traits (i.e. levels of ATP, ADP, IMP, creatine phosphate, glycogen content, $\mathrm{pH}$, and drip loss). Resting reverses the expression changes induced by exercise. Interestingly, overreaction of the reversal was observed for several proteins. The expression levels of several proteins both react to exercise and resting, and they are also associated with meat quality traits. This makes them very interesting biomarkers to determine and predict the optimal length of the resting time at the slaughterhouse after transport to the slaughterhouse, especially since resting too short shows reduced meat quality due to the transport stress, and resting too long may affect meat quality due to overreaction of the proteome to resting. The results also suggest that the optimal resting time varies according to the meat quality traits considered. Using these biomarkers may direct the carcasses to different market segments with different consumer expectations or processing industry requirements.

\subsection{Prenatal muscle tissue development related to post-mortem meat quality}

Muscle fibre numbers are important for meat quality characteristics. High muscularity can be achieved by muscle fibre hypertrophy or by muscle fibre hyperplasia. High muscle fibre hypertrophy like in Pietrain pigs often results in whiter meat (because type II fibres are thicker and require less vascularization than type I fibres) with higher drip loss and less IMF (Reviewed in Joo et al., 2013). High muscle fibre hyperplasia like in double-muscled cattle often results in more red meat with better organoleptic meat quality characteristics (Wegner et al., 2000). Mammalian muscle fibre formation is an exclusive prenatal process under strict genetic regulation (Wegner et al., 2000; te Pas and Soumillion, 2001; Rehfeldt et al., 2004; Stickland et al., 2004). Thus, muscle fibre formation is a prenatal process that regulates post-mortem meat quality. A major difference may be that the double-muscled cattle phenotype is caused by a mutation in the myostatin gene - a major (negative) muscle growth regulatory gene (McPherron et al., 1997; Grobet et al., 1998; Karim et al., 2000), while muscle fibre hypertrophy may be the result of the cooperation between many regulatory genes. A different mutation creating a regulatory miRNA site was found in the sheep myostatin gene generating an miRNA binding site in the myostatin mRNA, leading to down-regulation of the expression of the myostatin gene in the high-meatiness Texel sheep breed (Clop et al., 2006). However, no evidence exists for a mutation in the porcine myostatin gene (Stinckens et al., 2008). Therefore, we focused on the mechanism of muscle development and growth during prenatal pig development.

We studied the transcriptome expression in porcine longissimus dorsi muscles of Duroc pigs. The results showed that especially the primary wave of prenatal muscle fibre formation is strongly genetically regulated, while the secondary muscle fibre formation is associated with much less differential gene expression (te Pas et al., 2005; Cagnazzo et al., 2006). This is in agreement with the fact that the secondary muscle fibre formation can be regulated with feed, while the primary muscle fibre formation cannot be affected (Rehfeldt et al., 2004; Stickland et al., 2004; reviewed in Rehfeldt et al., 2011a, b). Interestingly, the number of differently expressed genes for energy metabolism suggest that proliferation of myoblasts prior to differentiation is accompanied by oxidative phosphorylation, while during differentiation (i.e. fusion into multinucleated muscle fibres) glycolysis seems to be of main importance (te Pas et al., 2005).

Interestingly, comparing Duroc and Pietrain data reveals that the expression of the myogenesis-related genes was greater in early Duroc embryos than in early Pietrain embryos, whereas the opposite was found in late embryos. These findings suggest that the primary myogenesis process is more intense in Duroc than in Pietrain embryos but that the secondary myogenesis that occurs later during gestation is more intense in Pietrain foetuses than in Duroc foetuses (Cagnazzo et al., 2006). These results were confirmed by Davoli et al. (2011) using PCR tests. Furthermore, the energy metabolism genes were expressed at a higher level in prenatal Pietrain pigs than in prenatal Duroc pigs, except at day 35 of gestation, when the opposite situation was found. Fatty acid metabolism genes were expressed at a higher level at early physiological stages in Duroc embryos than in Pietrain embryos. These results suggest that decades of breeding for high growth rate and lean muscularity in pigs (Merks, 2000) induced changes in the genetic regulation of the underlying biological processes that already are evident in the early embryo, including mechanisms that regulate post-mortem processes.

The next polymorphisms in the loci of 10 of the prenatal differentially expressed genes were studied for genetic association with meat quality traits (Wimmers et al., 2007) in 1700 performance-tested fattening pigs of commercial purebred and crossbred herds of Duroc, Pietrain, Pietrain $\times$ (Landrace $\times$ Large White $)$, and Duroc $\times($ Landrace $\times$ Large White $)$ as well as in an experimental F2 population based on a reciprocal cross of Duroc and Pietrain. This also served as a validation of the results in genetically different populations. Nine of the genes showed association with meat-quality and carcass traits, including meat colour, $\mathrm{pH}$, and conductivity of loin $24 \mathrm{~h}$ post-mortem. 
However, none of the genes showed significant associations for a particular trait across all populations indicating the breed-specific aspects of the results.

\section{Integration of knowledge and potential uses of the biomarkers}

These examples show that there is overwhelming evidence for the concept of biomarkers detecting and predicting meat quality traits in diverse pork production chains. While indeed breed-specific effects were reported, validation studies in different breeds also indicated evidence for biomarkers over breeds and production systems. This shows the commercial potential for biomarkers.

Different omics technologies may identify different biomarkers. However, biological mechanisms underlying the observed biomarkers may identify even more potential biomarkers. The underlying reason for the differences between the analyses may be related to the complex nature of the traits under investigation. For high reliability of the results it may be important to measure many genes/proteins/metabolites at the same time and conclude about the predictive capacity of the biomarker on the combined analysis of all measured genes/proteins/metabolites together. This will bring the biomarker research into the systems biology discipline (Woelders et al., 2011; te Pas et al., 2011b). A complexing factor is that all omics and phenotype data need to be measured in the same animals at the same moment in life (or after slaughter). This requires both high financial and technical resources. At present no example of this in livestock science exists. Orozco et al. (2015) provide a good example comparing 90 laboratory strains of mice, investigating genome-wide genotype (genetics), genome-wide methylation patterns (epigenetics), transcriptomics, proteomics, and metabolomic patterns. The analysis integrates all data together, clearly showing that genetic analysis using GWAS (genome-wide association study) provides only part of the genomic effect. A clear bidirectional interaction is shown for epigenetic effects. The resulting phenotypes of the 90 mouse strains also strongly relates to regulation at the transcriptomic, proteomic, and metabolomic levels. This study shows both the power and the necessity of integrated analysis to understand complex traits such as meat quality. Therefore, we argue that the future direction to understand meat quality and develop meaningful biomarkers needs to aim in this direction.

Automation will be required to reduce the costs until it is economically feasible to measure and use the biomarkers. The SNP-chip methods available now for genotyping of commercial herds are a good example of this. While measuring all SNPs was in the past too time-consuming and costly for commercial applications, genomic selection now applies SNP-chip data to whole cattle breeding herds, reducing generation time and reducing costs of testing herds (Calus and
Veerkamp, 2007). Robotized carcass direction systems exist, but the software interface between biomarker detection apparatus and the robotized slaughterhouse system still needs to be written. In the end, automated detection systems will reduce the costs of biomarker measurements because they do not require a specialized laboratory and specialized skilled laboratory personnel.

Biomarkers may also be important for research aiming to change traits. Intervention studies changing, for example, the environment of the animal (differing from feed composition to animal management at the farm and the transportation periods of piglets to the fattener and slaughter pigs to the slaughterhouse) can use biomarkers to predict the potential reaction of the animal to the intervention and to monitor changes during the intervention, aiming to reduce or reverse the adverse effects. Before the study starts, the biomarkers need to be well chosen and the method of detection determined. Breed-specific effects may be important for the choice, including the period of life of the intervention.

\subsection{Commercialization of biomarkers}

Pork is produced in chains integrating the activity of many industries: from breeders and producers with interaction with the feed producers to transporters and slaughterhouses, and either directly from the slaughterhouse to the retail sector or via the processing industry, and, finally, the consumers, which are not only the end users but also the ones who decide what quality really is. All industries in the chain should recognize that the consumers in the end judge the overall contributions that determine quality of the final product. Therefore, each industry should optimize its process in conjunction with all the other participants in the production chain. A combination of well-chosen biomarkers may help the industries to coordinate all production processes and to inform the other industries in the chain about their contributions to high meat quality. This will form an integrated production chain from which all participating actors will profit (te Pas et al., 2011a). This requires that all industries participating in the meat production chain accept the concept of biomarkers and cooperate in sharing of data among all partners, enabling integrated analysis of the data with only one goal in mind: producing meat with the highest possible quality for consumers.

Data availability. No data sets were used in this article.

Author contributions. MFWTP wrote the first draft of the manuscript and DL and NO added text of specific paragraphs. 
Competing interests. The authors declare that they have no conflict of interest.

Edited by: Steffen Maak

Reviewed by: two anonymous referees

\section{References}

Bailey, A. J. and Light, N. D.: Connective tissue in meat and meat products, Barking, England, Elsevier Applied Science, 637.51 BAIc, xiii, 355 pp., ISBN-10: 1-85166-284-7, 1989.

Benjamini, Y. and Hochberg, Y.: Controlling the false discovery rate: A practical and powerful approach to multiple testing, J. Roy. Stat. Soc. B, 57, 289-300, 1995.

Bonneau, M. and Lebret, B.: Production systems and influence on eating quality of pork, Meat Sci., 84, 293-300, 2010.

Cagnazzo, M., te Pas, M. F. W., Priem, J., de Wit, A., Pool, M. H., Davoli, R., and Russo, V.: Comparison of prenatal muscle tissue expression profiles of two pig breeds differing in muscle characteristics, J. Anim. Sci., 84, 1-10, 2006.

Callow, E. H.: The actions of salts and other substances used in the curing of bacon and ham, Br. J. Nutr., 1, 269-274, 1947.

Calus, M. P. L. and Veerkamp, R. F.: Accuracy of breeding values when using and ignoring the polygenic effect in genomic breeding value estimation with a marker density of one SNP per cM, J. Anim. Breed. Genet., 124, 362-368, 2007.

Cameron, N. D. and Enser, M. B.: Fatty acid composition of lipid in Longissimus dorsi muscle of Duroc and British Landrace pigs and its relationship with eating quality, Meat Sci., 29, 295-307, 1991.

Ciobanu, D., Bastiaansen, J., Malek, M., Woollard, J. J., Plastow, G., and Rothschild, M.: Evidence for New Alleles in the Protein Kinase Adenosine Monophosphate-Activated 3-Subunit Gene Associated With Low Glycogen Content in Pig Skeletal Muscle and Improved Meat Quality, Genetics, 159, 1151-1162, 2001.

Clop, A., Marcq, F., Takeda, H., Pirottin, D., Tordoir, X., Bibé, B., Bouix, J., Caiment, F., Elsen, J.-M., Eychenne, F., Larzul, C., Laville, E., Meish, F., Milenkovic, D., Tobin, J., Charlier, C., and Georges, M.: A mutation creating a potential illegitimate microRNA target site in the myostatin gene affects muscularity in sheep, Nature Genet., 38, 813-818, 2006.

Damon, M., Louveau, I., Lefaucheur, L., Lebret, B., Vincent, A., Leroy, P., Sanchez, M. P., Herpin, P., and Gondret, F.: Number of intramuscular adipocytes and fatty acid binding protein- 4 content are significant indicators of intramuscular fat level in crossbred Large White X Duroc pigs, J. Anim. Sci., 84, 1083-1092, 2006.

Damon, M., Wyszynska-Koko, J., Vincent, A., Hérault, F., and Lebret, B.: Comparison of Muscle Transcriptome between Pigs with Divergent Meat Quality Phenotypes Identifies Genes Related to Muscle Metabolism and Structure, PLoS ONE, 7, e33763, https://doi.org/10.1371/journal.pone.0033763, 2012.

Damon, M., Denieul, K., Vincent, A., Bonhomme, N., WyszynskaKoko, J., and Lebret, B.: Associations between muscle gene expression pattern and technological and sensory meat traits highlight new biomarkers for pork quality assessment, Meat Sci., 95, 744-754, 2013.

Davoli, R., Braglia, S., Russo, V., Varona, L., and te Pas, M. F. W.: Expression profiling of functional genes in prenatal skeletal mus- cle tissue in Duroc and Pietrain pigs, J. Anim. Breed. Genet., 128, 15-27, 2011.

Fernandez, X., Monin, G., Talmant, A., Mourot, J., and Lebret, B.: Influence of intramuscular fat content on the quality of pig meat -2 . Consumer acceptability of $\mathrm{m}$. longissimus lumborum, Meat Sci., 53, 67-72, 1999.

Gammeltoft, S.: Insulin receptors: binding kinetics and structurefunction relationship of insulin, Physiol. Rev., 64, 1321-1378, 1984.

Gandemer, G.: Lipids in muscles and adipose tissues, changes during processing and sensory properties of meat products, Meat Sci., 62, 309-321, 2002.

Gerbens, F., Rettenberger, G., Lenstra, J. A., Veerkamp, J. H., and te Pas, M. F. W.: Characterization, chromosomal localization, and genetic variation of the porcine heart fatty acid binding protein gene, Mamm. Gen., 8, 328-332, 1997.

Gerbens, F., Jansen, A., Van Erp, A. J. M., Harders, F., Meuwissen, T. H. E., Rettenberger, G., Veerkamp, J. H., and Te Pas, M. F. W.: The adipocyte fatty acid-binding protein locus: characterization and association with intramuscular fat content in pigs, Mamm. Gen., 9, 1022-1026, 1998.

Gerbens, F., van Erp, A. J. M., Harders, F. L., Verburg, F. J., Meuwissen, T. H. E., Veerkamp, J. H., and te Pas, M. F. W.: Effect of genetic variants of the heart fatty acid-binding protein gene on intramuscular fat and performance traits in pigs, J. Anim. Sci., 77, 846-852, 1999.

Gerbens, F., de Koning, D. J., Harders, F. L., Meuwissen, T. H. E., Janss, L. L. G., Groenen, M. A. M., Veerkamp, J. H., van Arendonk, J. A. M., and te Pas, M. F. W.: The effect of genetic variants of adipocyte and heart fatty acid-binding protein on intramuscular fat and backfat content in Meishan crossbred pigs, J. Anim. Sci., 78, 552-559, 2000.

Gerbens, F., Verburg, F. J., van Moerkerk, H. T. B., Engel, B., Buist, W., Veerkamp, J. H., and te Pas, M. F. W.: Associations of heart and adipocyte fatty acid-binding protein gene expression with intramuscular fat content in pigs, J. Anim. Sci., 79, 347-354, 2001.

Goodsaid, F. and Frueh, F.: Biomarker Qualification Pilot Process at the US Food and Drug Administration, Am. Assoc. Pharm. Scientists J., 9, E105-E108, 2007.

Grandin, T.: The effect of stress on livestock and meat quality prior to and during slaughter, Int. J. Study Anim. Probl., 1, 313-337, 1980.

Grobet, L., Poncelet, D., Royo, L. J., Brouwers, B., Pirottin, D., Michaux, C., Ménissier, F., Zanotti, M., Dunner, S., and Georges, M.: Molecular definition of an allelic series of mutations disrupting the myostatin function and causing double muscling in cattle, Mamm. Gen., 9, 210-213, 1998.

Henckel, P., Karlsson, A., Oksbjerg, N., and Søholm Petersen, J.: Control of post mortem $\mathrm{pH}$ decrease in pig muscles: experimental design and testing of animal models, Meat Sci., 55, 131-138, 2000.

Herault, F., Vincent, A., Dameron, O., Le Roy, P., Cherel, P., and Damon, M.: The Longissimus and Semimembranosus Muscles Display Marked Differences in Their Gene Expression Profiles in Pig, PloS ONE, 9, e96491, https://doi.org/10.1371/journal.pone.0096491 2014.

Honikel, K. O.: Reference methods for the assessment of physical characteristics of meat, Meat Sci., 49, 447-457, 1998. 
Hovenier, R., Kanis, E., and Verhoeven, J. A.: Repeatability of taste panel tenderness scores and their relationships to objective pig meat quality traits, J. Anim. Sci., 71, 2018-2025, 1993.

Joo, S. T., Kim, G. D., Hwang, Y. H., and Ryu, Y. C.: Control of fresh meat quality through manipulation of muscle fibre characteristics, Meat Sci., 95, 828-836, 2013.

Kadonaga, J. T.: Eukaryotic Transcription: An Interlaced Network of Transcription Factors and Chromatin-Modifying Machines, Cell, 92, 307-313, 1998.

Karim, L., Coppieters, W., Grobet, L., Valentini, A., and Georges, M.: Convenient genotyping of six myostatin mutations causing double-muscling in cattle using a multiplex oligonucleotide ligation assay, Anim. Genet., 31, 396-399, 2000.

Kuchenmeister, U., Kuhn, G., Stabenow, B., and Ender, K.: The effect of experimental stress on sarcoplasmic reticulum $\mathrm{Ca} 2+$ transport and meat quality in pig muscle, Meat Sci., 61, 375-380, 2002.

Kuchenmeister, U., Kuhn, G., and Ender, K.: Preslaughter handling of pigs and the effect on heart rate, meat quality, including tenderness, and sarcoplasmic reticulum $\mathrm{Ca} 2+$ transport, Meat Sci., 71, 690-695, 2005.

Kukoyi, E. A., Addis, P. B., McGrath, C. J., Rempel, W. E., and Martin, F. B.: Porcine Stress Syndrome and Postmortem Muscle Characteristics of Two Purebreds and Three Specific Terminal Crosses, J. Anim. Sci., 52, 278-284, 1981.

Lebret, B., Denieul, K., and Damon, M.: Muscle transcriptome profiles highlight biomarkers of pig production system and high meat quality, Proc. 59th International Congress of Meat Science and Technology, 18-23 August, Izmir, Turkey, no. O13, p. 33, 2013a.

Lebret, B., Denieul, K., Vincent, A., Bonhomme, N., WyszynskaKoko, J., Kristensen, L., Young, J. F., and Damon, M.: Identification by transcriptomics of biomarkers of pork quality, J. Recherche Porc., 45, 97-102, 2013b.

Lebret, B., Vincent, A., and Faure, J.: Muscle biomarkers to differentiate pork quality categories based on industry and consumer demands, 65th Ann. Meet. Eur. Assoc. Anim. Prod., 25-29 August, Copenhagen, Denmark, p. 440, 2014.

Lebret, B., Ecolan, P., Bonhomme, N., Méteau, K., and Prunier, A.: Influence of production system in local and conventional pig breeds on stress indicators at slaughter, muscle and meat traits and pork eating quality, Animal, 9, 1404-1413, 2015.

Lebret, B. and Picard, B.: The main components of carcasses and meat quality in various animal species, in: Special issue of Le muscle et la viande, edited by: Picard, B. and Lebret, B., INRA Productions Animales, 28, 93-98, 2015.

Lefaucheur, L.: A second look into fibre typing - Relation to meat quality, Meat Sci., 84, 257-270, 2010.

Li, J.-H., Liu, S., Zhou, H., Qu, L.-H., and Yang, J.-H.: StarBase v2.0: decoding miRNA-ceRNA, miRNA-ncRNA and proteinRNA interaction networks from large-scale CLIP-Seq data, Nucleic Acid. Res., 42 , 1-6, https://doi.org/10.1093/nar/gkt1248, 2013.

Listrat, A., Lebret, B., Louveau, I., Astruc, T., Bonnet, M., Lefaucheur, L., Picard, B., and Bugeon, J.: How do muscle structure and composition determine the meat and flesh quality?, Sci. World J., 3182746, https://doi.org/10.1155/2016/3182746, 2016.

Mach, N., Keuning, E., Kruijt, L., Hortós, M., Arnau, J., and te Pas, M. F. W.: Comparative proteomic profiling of two muscles from five divergent pig breeds using SELDI-TOF proteomics technology, J. Anim. Sci., 88, 1522-1534, 2010.

Marcos, B., Gou, P., Serra, X., Guàrdia, M. D., Zongyuan, Z., Hortós, M., Mach, N., te Pas, M. F. W., Keuning, E., Kruijt, L., Font i Furnols, M., and Arnau, J.: Analysis of raw hams using SELDI-TOF-MS to predict the final quality of dry-cured hams, Meat Sci., 93, 233-239, 2013a.

Marcos, B., Gou, P., Guàrdia, M. D., Hortós, M., Colleo, M., Mach, N., te Pas, M. F. W., Keuning, E., Kruijt, L., Tibau, J., Gispert, M., and Arnau, J.: Surface-Enhanced Laser Desorption/Ionisation Time-of-Flight Mass Spectrometry: a Fast Method to Assess Pork Quality, Meat Sci., 95, 688-693, 2013 b.

Meats and Sausages: available at: http://www.meatsandsausages. com/sausage-making/curing/methods, last access: 14 June 2017.

McPherron, A. C., Lawler, A. M., and Lee, S. J.: Regulation of skeletal muscle mass in mice by a new TGF- $\beta$ superfamily member, Nature, 387, 83-90, 1997.

Merks, J. W. M.: One century of genetic changes in pigs and the future needs, in: The Challenge of Genetic Change in Animal Production, edited by: Hill, W. G., Bishop, S. C., McGuirk, B., McKay, J. C., Simm, G., and Webb, A. J., Occasional Publication No. 27, Brit. Soc. Anim. Sci., Edinburgh, UK, 8-19, 2000.

Milan, D., Jeon, J.-T., Looft, C., Amarger, V., Robic, A., Thelander, M., Rogel-Gaillard, C., Paul, S., Iannuccelli, N., Rask, L., Ronne, H., Lundström, K., Reinsch, N., Gellin, J., Kalm, E., Le Roy, P., Chardon, P., and Andersson, L.: A Mutation in PRKAG3 Associated with Excess Glycogen Content in Pig Skeletal Muscle, Science, 288, 1248-1251, 2000.

Ngapo, T. M. and Gariépy, C.: Factors Affecting the Eating Quality of Pork, Crit. Rev. Food Sci. Nutr., 48, 599-633, 2008.

Orozco, L. D., Morselli, M., Rubbi, L., Guo, W., Go, J., Shi, H., Lopez, D., Furlotte, N. A., Bennett, B. J., Farber, C. R., Ghazalpour, A., Zhang, M. Q., Bahous, R., Rozen, R., Lusis, A. J., and Pellegrini, M.: Epigenome-Wide Association of Liver Methylation Patterns and Complex Metabolic Traits in Mice, Cell Metab., 21, 905-917, 2015.

Phillips, D. I. W., Clark, P. M., Hales, C. N., and Osmond, C.: Understanding oral glucose tolerance: comparison of glucose or insulin measurements during the oral glucose tolerance test with specific measurements of insulin resistance and insulin secretion, Diab. Med., 11, 286-292, 1994.

Picard, B., Lefèvre, F., and Lebret, B.: Meat and fish flesh quality improvement with proteomic applications, Anim. Front., 2, 18$25,2012$.

Picard, B., Lebret, B., Cassar-Malek, I., Liaubet, L., Berri, C., Le Bihan-Duval, E., Hocquette, J. F., and Renand G.: Recent advances omic technologies for meat quality management, Meat Sci., 109, 18-26, 2015.

Pierzchala, M., Hoekman, A. J. W., Urbanski, P., Kruijt, L., Kristensen, L., Young, J. F., and te Pas, M. F. W.: Validation of biomarkers for loin meat quality (m. longissimus) of pigs, J. Anim. Breed. Genet., 131, 258-270, 2014.

Purslow, P. P.: Intramuscular connective tissue and its role in meat quality, Meat Sci., 70, 435-447, 2005.

Rehfeldt, C., Fiedler, I., and Stickland, N. C.: Number and size of muscle fibres in relation to meat production, in: Muscle Development of Livestock Animals - Physiology, Genetics, and Meat Quality, edited by: te Pas, M. F. W., Everts, M. E., and Haags- 
man, H. P., CABI publishers, Wallingford, Oxfordshire, UK, 138, 2004.

Rehfeldt, C., Te Pas, M. F. W., Wimmers, K., Brameld, J. M., Nissen, P. M., Berri, C., Valente, L. M. P., Power, D., Picard, B., Stickland, N. C., and Oksbjerg, N.: Advances in research on the prenatal development of skeletal muscle in animals in relation to the quality of muscle-based food. I. Regulation of myogenesis and environmental impact, Animal, 5, 703-717, 2011a.

Rehfeldt, C., Te Pas, M. F. W., Wimmers, K., Brameld, J. M., Nissen, P. M., Berri, C., Valente, L. M. P., Power, D., Picard, B., Stickland, N. C., and Oksbjerg, N.: Advances in research on the prenatal development of skeletal muscle in animals in relation to the quality of muscle-based food. II. Genetic factors related to animal performance and advances in methodology, Animal, 5, 718-730, 2011b.

Rosenvold, K., Petersen, J. S., Lwerke, H. N., Jensen, S. K., Therkildsen, M., Karlsson, A. H., Møller, H. S., and Andersen, H. J.: Muscle glycogen stores and meat quality as affected by strategic finishing feeding of slaughter pigs, J. Anim. Sci., 79, 382-391, 2001.

Schäfer, A., Rosenvold, K., Andersen H. J., and Henckel, P.: Physiological and structural events post mortem of importance for drip loss in pork, Meat Sci., 61, 355-366, 2002.

Stickland, N. C., Bayol, S., Ashton, C., and Rehfeldt, C.: Manipulation of muscle fibre number during prenatal development, in: Muscle Development of Livestock Animals - Physiology, Genetics, and Meat Quality, edited by: te Pas, M. F. W., Everts, M. E., and Haagsman, H. P., CABI publishers, Wallingford, Oxfordshire, UK, 69-82, 2004.

Stinckens, A., Luyten, T., Bijttebier, J., Van den Maagdenberg, K., Dieltiens, D., Janssens, S., De Smet, S., Georges, M., and Buys, N.: Characterization of the complete porcine MSTN gene and expression levels in pig breeds differing in muscularity, Anim. Genet., 39, 586-596, 2008.

Støier, S., Aaslyng, M. D., Olsen, E. V., and Henckel, P.: The effect of stress during lairage and stunning on muscle metabolism and drip loss in Danish pork, Meat Sci., 59, 127-131, 2001.

te Pas, M. F. W. and Hoekman, A. J. W.: Developing biomarkers to improve, detect and monitor high pork quality, in: Pork: nutrition, consumption and Health, edited by: Hendrks, B. P., Agricultural Res. Updates 3, e-book, ISBN-13: 978-1-62100-380-9, 2012.

te Pas, M. F. W. and Soumillion, A.: The use of physiologic and functional genomic information of the regulation of the determination of skeletal muscle mass in livestock breeding strategies to enhance meat production, Curr. Genom., 2, 285-304, 2001.

te Pas, M. F. W., de Wit, A., Priem, J., Cagnazzo, M., Davoli, R., Russo, V., and Pool, M. H.: Transcriptome expression profiles in prenatal pigs in relation to myogenesis, J. Muscle Res. Cell Motil., 26, 157-165, 2005.

te Pas, M. F. W., Pool, M. H., Hulsegge, I., and Janss, L. L. G.: Analysis of the differential transcriptome expression profiles during prenatal muscle tissue development, Arch. Anim. Breed., SI 49, $110-115,2006$.

te Pas, M. F. W., Hulsegge, I., and Pool, M. H.: Pathway analysis: combining microarray data and physiological data to study myogenesis, Arch. Anim. Breed., SI 50, 27-31, 2007a.

te Pas, M. F. W., Hulsegge, I., Pool, M. H., Coster, A., Heuven, H. H., and Janss, L. L. G.: Biochemical pathways analysis of microarray results: regulation of myogenesis, BMC Devel. Biol., 7, 1-15, https://doi.org/10.1186/1471-213X-7-66, 2007b.

te Pas, M. F. W., Jansen, J., Broekman, K. C. J. A., Reimert, H., and Heuven, H. C. M.: Post mortem proteome degradation profiles of longissimus muscle in Yorkshire and Duroc pigs and its relationship with pork quality traits, Meat Sci., 83, 744-751, 2009.

te Pas, M. F. W., Keuning, E., Hulsegge, B., Hoving-Bolink, A. H., Evans, G., and Mulder, H. A.: Longissimus muscle transcriptome profiles related to carcass and meat quality traits in fresh meat Pietrain carcasses, J. Anim. Sci., 88, 4044-4055, 2010.

te Pas, M. F. W., Hoekman, A. J. W., and Smits, M. A.: Biomarkers as management tools for the pork production chain industries, J. Chain Netw. Sci., 11, 155-166, 2011 a.

te Pas, M. F. W., Woelders, H., and Bannink, A. (Eds.): Systems Biology and Livestock Science, John Wiley and Sons, Chichester, UK, ISBN-10: 0-8138-1174-0, ISBN-13: 978-0-8138-1174-1, 2011b.

te Pas, M. F. W., Keuning, E., van de Wiel, D. J. M., Young, J. F., Oksbjerg, N., and Kruijt, L.: Proteome Profiles of Longissimus and Biceps femoris Porcine Muscles Related to Exercise and Resting, J. Life Sci., 5, 598-608, 2011c.

te Pas, M. F. W., Kruijt, L., Pierzchala, M., Crump, R. E., Boeren, S., Keuning, E., Hoving-Bolink, R. A., Hortós, M., Gispert, M., Arnau, J., Diestre, A., and Mulder, H. A.: Identification of proteomic biomarkers in M. Longissimus dorsi as potential predictors of pork quality, Meat Sci., 95, 679-687, 2013.

Tisch, R. and McDevitt, H.: Insulin dependent diabetes mellitus, Cell., 85, 291-297, 1996.

Toldra, F., Flores, M., and Sanz, Y.: Dry-cured ham flavor: enzymatic generation and process influence, Food Chem., 59, 523530, 1997.

Toldra, F. and Flores, M.: The Role of Muscle Proteases and Lipases in Flavor Development During the Processing of DryCured Ham, Crit. Rev. Food Sci. Nutr., 38, 331-352, 1998.

Van der Wal, P. G., Engel, B., and Reimert, H. G. M.: The effect of stress, applied immediately before stunning, on pork quality, Meat Sci., 53, 101-106, 1999.

Warriss, P. D.: Meat science: an introductory text, 2nd edition, CABI, Wallingford, UK, 234 pp., 2010.

Wegner, J., Albrecht, E., Fiedler, I., Teuscher, F., Papstein, H. J., and Ender, K.: Growth- and breed-related changes of muscle fibre characteristics in cattle, J. Anim. Sci., 78, 1485-1496, 2000.

Wierbicki, E. and Howker, J. J.: Effect of salt, phosphates and other curing ingredients on shrinkage of lean pork meat and quality of smoked processed ham, J. Food Sci., 41, 1116-1121, 1976.

Wilson, K. J. and De la Vega, E.: The potential of microarrays to assist shrimp breeding and production: a review, Austr. J. Exp. Agric., 45, 901-911, 2005.

Wimmers, K., Murani, E., te Pas, M. F. W., Chang, K. C., Davoli, R., Merks, J. W. M., Henne, H., da Costa, N., Harlizius, B., Schellander, K., Braglia, S., de Wit, A. A. C., Cagnazzo, M., Fontanesi, L., Prins, D., and Ponsuksili, S.: Associations of functional candidate genes derived from gene expression profiles of prenatal porcine muscle tissue with meat quality and carcass traits, Anim. Genet., 38, 474-484, 2007.

Woelders, H., te Pas, M. F. W., Bannink, A., Veerkamp, R. F., and Smits, M. A.: Systems biology in animal sciences, Animal, 5, 1036-1047, 2011. 
Young, J. F., Bertram, H. C., and Oksbjerg, N.: Rest before slaughter ameliorates pre-slaughter stress-induced increased drip loss but not stress-induced increase in the toughness of pork, Meat Sci., 83, 634-641, 2009.
Zhou, G. H. and Zhao, G. M.: Biochemical changes during processing of traditional Jinhua ham, Meat Sci., 77, 114-120, 2007. 